\title{
21. On the Spaces with normal conformal Connexions whose Groups of Holonomy fix a Point or a Hypersphere. III.
}

\author{
By \\ Shigeo SASAKI. \\ (Received December 19, 1942.)
}

\section{Introduction.}

In my previous paper [1] I have proved that "if the group of holonomy $H_{n}$ of a space with a normal conformal connexion $C_{n}$ fixes a point or a hypersphere in the annexed Möbius space, $C_{n}$ is a conformal space corresponding to a set of Riemann spaces conformal to each other and containing in them at least an Einstein space with vanishing or non-vanishing scalar curvature according as $H_{n}$ fixes a point or a hypersphere. The converse is also true."

This theorem is very important, indeed, many of the theorems obtained by E. Cartan and H. W. Brinkmann can be deduced, in virtue of the last theorem, by purely geometrical reasonings. In the present paper, I will prove, again making use of the theorem, that there exist representations for Einstein spaces analogous to the Poincaré's representations in Non-Euclidean geometries.

\section{$\S 1$. The Poincaré's representations in tangent spaces.}

Let us consider a space with a normal conformal connexion $C_{n}$ whose group of holonomy $H_{n}$ fixes a hypersphere (not a point) $S$. To the space $C_{n}$ there corresponds a set of Riemann spaces conformal to each other, one of them being an Einstein space with a non-vanishing scalar curvature (I. the fundamental theorem). We shall use as a system of reference for $C_{n}$ the Veblen's reperes determined by the fundamental tensor $g_{j k}$ of the Einstein space. Then the conformal connexion of $C_{n}$ can be given by the following formulas:

The cost of this research has been defrayed from the Scientific Research Expenditure of the Department of Education. 


$$
\begin{array}{ll}
d R_{0}= & d x^{i} R_{\mathbf{i}} \\
d R_{j}=c g_{j k} d x^{k} R_{0}+\{\underset{j}{j}\} d x^{k} R_{i}+g_{j k} d x^{k} R_{\infty} \\
d R_{\infty}= & c d x^{i} R_{\mathbf{i}} .
\end{array}
$$

Now, let $x^{i}(s)$ be a conformal circle $C$ in $C_{n}$, denoting by $s$ its arc-length. If we develop the curve $C$ in the tangent Möbius space $M_{n}\left(P_{0}\right)$ at a point $P_{0}$ of $C$, the image $C^{*}$ of $C$ is expressible, choosing a suitable projective parameter, as follows in virtue of (1):

$$
p^{*}=\left(1+\frac{t^{2}}{4} g_{j k} n^{j} n^{k}\right) R_{0}+\left(\frac{d x^{i}}{d s} t+\frac{t^{2}}{2} n^{i}\right) R_{i}+\frac{t^{2}}{2} R_{\infty},
$$

where we have put

$$
n^{i}=\frac{d^{2} x^{i}}{d s^{2}}+\{\underset{j k}{i}\} \frac{d x^{j}}{d s} \frac{d x^{k}}{d s} .
$$

The points of intersection of the circle $C^{*}$ and the invariant hypersphere $S \equiv R_{\infty}-c R_{0}$ are those having as their projective parameters the solutions of the following equation:

$$
\left(\frac{c}{2}-\frac{1}{4} g_{j k} n^{j} n^{k}\right) t^{2}=1 .
$$

In order that $C^{*}$ cuts $S$ ortogonally, $P^{*}$ must belong to the pencil of hyperspheres determined by $S$ and $\frac{d P^{*}}{d t}$, in which $t$ is replaced by a solution $t$ of (2). This shows us immediately that $n^{i}$ is proportional to $\frac{d x^{i}}{d s}$. Accordingly, the functions $x^{i}(s)$ must be solutions of the following equations :

$$
\frac{d^{2} x^{i}}{d s^{2}}+\{\underset{j k}{i}\} \frac{d x^{j}}{d s} \frac{d x^{k}}{d s}=0 .
$$

Then the values of $t$ for the points of intersection of $C^{*}$ and $S$, are given by

$$
t= \pm \sqrt{\frac{2}{c}} .
$$

Our reasoning is also applicable for the case $c=0$, i.e. for any space with a normal conformal connexion whose group of holonomy fixes a point. Hence we get the following

Theorem 1. If the group of holonomy of a space with a normal conformal connexion $C_{n}$ fixes a hypersphere (or a point) $S$, any conformal 
circle, having a circle orthogonal to the invariant hypersphere (or a circle passing through the invariant point) $S$ as its image, is a geodesic of the Einstein space corresponding to the invariant hypersphere (or point) S. The converse is also true.

Corollary. Suppose that the group of holonomy of a space with a normal conformal connexion $C_{n}$ fixes a hypersphere (or a point) $S$. If the Einstein space corresponding to $S$ admits a totally geodesic hypersurface $U$, the hypersphere $U^{*}$, which is the image of $U$ in $M_{n}$, cuts the invariant hypersphere $S$ orthogonally (or pass through the point $S$ ).

Now, between the proiective parameter and the arc-length of a curve there exists a relation

$$
\{t, s\}=\frac{R}{2 n(n-1)}, \quad\left(\because c=\frac{-R}{2 n(n-1)}\right)
$$

where $\{t, s\}$ denotes the Schwarzian derivative [2].

In case $R>0$, a particular solution of (3) is given by

$$
t=\frac{2}{k} \tan \frac{k s}{2}, \quad k=\sqrt{\frac{R}{n(n-1)}}
$$

and the other solutions are obtainable from it by applying linear fractional transformations. In considering only double ratios for sets of four values of $t$ we may take without any loss of generality the solution $t$, given by the last equation, instead of the general solution of (3). By Theorem 1 , any geodesic $C$ in Einstein space is developed to a circle $C^{*}$ cutting orthogonally the imaginary invariant hyperphere $S$. If we denote the double ratio of points $P_{0}(s=0, t=0), P_{s}(s=s, t=t)$ and the points, where $C^{*}$ cuts $S\left(t= \pm \frac{2 i}{k}\right)$ by $d$, we get

$$
t=\frac{2 i}{k} \frac{d-1}{d+1} .
$$

Eliminating $t$ from the last two equations we get

$$
s=\frac{i}{k} \log d
$$

In case $R<0$, one of the solutions of (3) is given by

$$
t=\frac{2}{k} \tanh \frac{k s}{2}, \quad k=\sqrt{\frac{-R}{n(n-1)}} .
$$


Applying the same reasoning as above we get

$$
t=\frac{2}{k} \frac{d-1}{d+1}
$$

and

$$
s=\frac{1}{k} \log d .
$$

Hence we obtain the following

Theorem 2. The distance $s$ between two points $P_{0}$ and $P_{s}$ on $a$ geodesic $C$ of an Einstein space with non-vanishing scalar curvature is equal to $\sqrt{\frac{n(n-1)}{-R}}$ times the natural logarithm of the double ratio determined by points $P_{0}^{*}, P_{s}^{*}$ and the points where the development $C^{*}$ of $C$ in a tangent Möbius space of the space with a normal conformal connexion corresponding to the set of Riemann spaces conformal to each other and containing in them the given Einstein space cut the invariant hypersphere $S$.

Theorems 1 and 2 show us that there exist representations for Einstein spaces similar to Poincaré's representations of Non-Euclidean geometries.

\section{$\S 2$. The Poincaré's representation in the underlying manifold.}

Let us consider a set of analytic Riemann spaces conformal to each other and construct a space with a normal conformal connexion $C_{n}$ corresponding to it. We assume here that if we draw an arbitrary conformal circle $C$ in $C_{n}, C$ can be extended to a point corresponding to any value of the projective parameter from an arbitrary point on $C$. The $C_{n}$ is said to be C-complete if it satisfies the above condition. In my previous paper II, I have shown that "if the group of holonomy of a C-complete space with a normal conformal connexion fixes a real hypersphere $S$, the $C_{n}$ admits of at least an umbilical hypersurface $U$ (II. Theorem 7)." The image of $U$ in the tangent space $M_{n}$ is the invariant hypersphere $S$.

Now, draw a geodesic $C$ in the sense of the Einstein space (whose existence is recognized by the fundamental theorem) and take two points $P_{0}$ and $P_{s}$ on $C . C$ is a conformal circle in $C_{n}$. If we consider the double ratio of the projective parameters of four points corresponding to $P_{0}, P_{s}$ and the points where the conformal circle $C$ cuts the umbilical hypersurface $U$, it is equal to the double ratio of the 
four points of their images i.e. $P_{0}^{*}, P_{s}^{*}$ and points where the circle $C^{*}$ cuts $S$. Hence we get the following

Theorem 3. Let $C_{n}$ be a C-complete space with a normal conformal connexion. If there exists an Einstein space with negative scalar curvature among Riemann spaces conformal to each other corresponding to $C_{n}, C_{n}$ admits of an umbilical hypersphere $U$. The distance between two points $P_{0}$ and $P_{s}$ on a geodesic $C$ of the Einstein space is equal to $\sqrt{\frac{n(n-1)}{-R}}$ times the natural logarithm of the double ratio of four values of projective parameters of $C$ corresponding to the points $P_{0}, P_{s}$ and the points where the geodesic $C$ cuts the umbilical hypersurface $U$.

Accordingly, there exists a representation analogous to the Poincare's representation of Non-Euclidean geometries for Einstein spaces with negative scalar curvature, not only for its tangent Möbius space but also for the underlying manifold itself.

We can conclude that the space with a normal conformal connexion, whose group of holonomy fixes a point or a hypersphere, plays the important rôle in the geometry of the conformal connexion just as space of constant curvature does in Riemann geometry. I think it is very important and interesting to study this space and many properties of Einstein spaces will be deduced by these methods in purely geometrical manners.

\section{References.}

[1] S. Sasaki, On the spaces with normal conformal connexions whose groups of holonomy fix a point or a hypersphere. I. II. Jap. Journ. Math. 18 (1943) pp. 615-622, 623-633.

[2] K. Yano-Y. Mutô, Sur la theorie des espaces à connexion conforme normale et la géométrie conforme des espaces de Riemann. Journ. Fac. Sci. Imp. Univ. Tokyo, Sect. I, vol. 4, pp. 117-167.

Mathematical Institute,

Tôhoku Imperial University. 